\title{
TEST TIME OPTIMIZATION BY REVISITING NOTES IN VLSI BIST TECHNIQUE
}

H. Sribhuvaneshwari

Research scholar, ECE Department, Kalasalingam Academy of Research and Education,

Krishnankoil, Tamilnadu, (India).

E-mail: havisriece@gmail.com ORCID: https://orcid.org/0000-0002-4804-4171

K. Suthendran

HoD/ IT Department, Kalasalingam Academy of Research and Education,

Krishnankoil, Tamilnadu, (India).

E-mail: k.suthendran@klu.ac.in ORCID: https://orcid.org/0000-0002-7030-4398

Recepción: 05/12/2019 Aceptación: 08/01/2020 Publicación: 23/03/2020

\section{Citación sugerida:}

Sribhuvaneshwari, H., y Suthendran, K. (2020). Test Time Optimization by Revisiting Notes in VLSI BIST Technique. 3 C Tecnología. Glosas de innovación aplicadas a la pyme. Edición Especial, Marzo 2020, 19-33. http://doi.org/10.17993/3ctecno.2020.specialissue4.19-33

\section{Suggested citation:}

Sribhuvaneshwari, H., \& Suthendran, K. (2020). Test Time Optimization by Revisiting Notes in VLSI BIST Technique. 3C Tecnología. Glosas de innovación aplicadas a la pyme. Edición Especial, Marzo 2020, 19-33. http://doi.org/10.17993/3ctecno.2020.specialissue4.19-33 


\section{ABSTRACT}

An effective method for test time minimization in Built In Self Test (BIST) using graph theory concept with revisiting of node is incorporated in this article. Here the shortest Hamiltonian path of ISCAS89 benchmark circuit s396 is taken as an example. Minimum spanning tree with revisiting nodes is applied for s386 circuit that optimizes the time cycle for testing. Result shows that minimum spanning tree with revisiting the nodes will reduce the time cycle without compromising the test quality. Hence an effective testing is achieved using graphical approach.

\section{KEYWORDS}

BIST, Shortest Hamiltonian path, Revisiting node, Test time. 


\section{INTRODUCTION}

In this super fast technical generation the growth of technology is massive in both technical and product aspect. Testing is an essential part which deals with the quality of the product before a microelectronic product is launched in the market where BIST is a testing scheme that is capable of finding faults in integrated circuits (ICs) to make faster testing at lessexpensive with low power constraints (Girard, Nicolici, \& Wen, 2010). It plays a vital role in electronic industry because a device that needs to be tested at higher level (levels being: Chip - board - system - system in field) costs 10 time (and possibly more) that of cost of testing it a lower level. Digital testing is declared as testing a digital circuit to validate that it performs the particular logic functions and in appropriate time. In case of VLSI testing, it is not of much concern as how many chips are binned as flawed; rather important is how many flawed chips are binned as normal. So, trade and industry expects "VLSI testing" is to result in an accuracy of perfect chips with its functionality. Optimized test time and scheming test power are contradictory targets and therefore optimization of testing for both attributes is challenging. This topic has been addressed in the recent literature (Nicolici \& Al-Hashimi, 2003; Sakurai \& Newton, 1990; Shanmugasundaram \& Agrawal, 2011; Shanmugasundaram \& Agrawal, 2012; Gogoi \& Kalita, 2014; Venkataramani, Sindia, \& Agrawal, 2014). The BIST vectors are speedy than ATE in terms of application time, thus follow-on improvement in test time with low power (Larsson, 2006). The test vector application time ratio between ATE and BIST is represented by " $a$ ". If $a=100$ than the application time of a vector in ATE is 100 times longer than the vector application of BIST (where $\alpha>1$ ). The total time required for test is equivalent to the addition of required number of time cycles to travel from source node to destination.

\section{PRIOR WORK}

A modern approach is introduced to minimize the test time for power constrained tests (Biswas, Das, \& Petriu, 2006; Das et al., 2008; Shanmugasundaram \& Agrawal, 2011, 2012) implements a monitor to observe the movement in the scan chain of a built-in self-test (BIST). According to the switching activity the test clock frequency varies from high to low both the parameters are inversely proportional i.e., test clock frequency raise if there is low switching activity in the scan chain else falls. This approach attains $20-50 \%$ reduction in test 
time of BIST circuits with a little area overhead. Reusable scan chains (Lai, Kung, \& Lin, 1993) and pattern overlapping (Zhou, Ye, Li, Wu, \& Ke, 2009; Bryant, 1986; Tehranipoor, Nourani, \& Chakrabarty, 2005; Chloupek, Novak, \& Jenicek, 2012; Chloupek, Novak, \& Jenicek, 2012; Alpert et al., 2018) eradicates unwanted scan chain operations using patterns that bear a resemblance to the previous pattern, so the number of scan shifting is minimized.

Hence high reduction is achieved on availability of such patterns. The single fixed order twisted ring-counter design proposed in (Tharakan \& Mathew, 2015) drops down the test application cycle with multiple programmable twisted-ring counters (PTRC). Huge number of unique test patterns based on the stipulation of reconfigurable run-time programmable multiple twisted-ring-counter is anticipated which is an on-chip test generation scheme. Spontaneous strategy is implemented in (Bhakthavatchalu, Krishnan, Vineeth, \& Devi, 2014) select the best possible seed and the quantity of the irregular test examples to be produced which reduces the testing time significantly. LFSR reseeding strategies proposed in (Kim \& Kang, 2006; Chandra \& Chakrabarty, 2003; Pathak \& Pathak, 2016) are broadly received in rationale BIST to improve fault perceptibility and abbreviate test application time for incorporated circuits.

Test comes about on ISCAS and expansive ITC circuits appear that the exhibited procedure can accomplish $100 \%$ fault scope with short test time by utilizing just $0.23-2.75 \%$ of inside nets. Test application time optimization in accumulator-based test-pattern generation is projected in (Magos, Voyiatzis, \& Tarnick, 2010; Voyiatzis, 2005, 2006, 2008; Manich, Garcia-Deiros, \& Figueras, 2007; Liang, Zhang, You, Li, \& Hosam, 2013). The problem of efficiently generating test patterns which is used in finding the shortest Hamiltonian path in an associated CUT's directed graph that results tremendously low demand for hardware.

Usage of accumulator structure is the better solution to the problem of minimizing the number of cycles needed for generating a set of deterministic test patterns in a novel test pattern generation. Further enhancement can be concentrated in terms of minimizing larger search space and the exact computation of the shortest Hamiltonian path in the testpattern graph. Revisiting nodes can reduce the test application time. 


\section{TEST PATTERN SELECTION}

All VLSI chips after the manufacturing process are applied for fault analysis, in such a case it is not possible of generating all the test vectors, at the same time different patterns detects the same fault which increases the complexity of test vector and its storage requirement. For C17 benchmark circuit the following six test patterns are high in terms of fault coverage which is shown in Table 1. These patterns are considered as node here.

$$
T=[T[1], T[2], T[3], T[4], T[5], T[6]]=T[0,11,14,17,28,31]
$$

Table 1. Test vector set of s396 circuit.

\begin{tabular}{|c|c|}
\hline Test Vector & Inputs [6:0] \\
\hline T1 & 0000101 \\
\hline T2 & 0000110 \\
\hline T3 & 0001011 \\
\hline T4 & 0001110 \\
\hline T5 & 0010101 \\
\hline T6 & 1010000 \\
\hline
\end{tabular}

The odd value sequence of $(31,3)$ is $\{0,3,6,9,12,15,18,21,24,27,30,2,5,8,11,14,17,20,23,26$ ,29,1,4,7,10,13,16,19,22,25,28,31 . Likewise it is preceded for all possible combination i.e., $(31, n)$. Here $\mathrm{n}$ is the odd numbers in-between ( 1 to 31$)$. In Table 2 decimal representation of the test vectors are given in first row, column and their location are given in $5 * 5$ matrix forms. Matrix size is equivalent to the number of test vectors in the test vector set of the concern circuit test pattern'00000' is negligible, so five test patterns are taken for calculation. $\left(2^{\mathrm{n}}+1\right) \&\left(2^{\mathrm{n}}+3\right)$ sequence i.e., $3,5,9,17 \& 5,7,11,19$ is calculated by means of Hamiltonian distance.

\section{DEFINITIONS}

Let $\mathrm{k}$ is the input to the particular circuit then $2^{\mathrm{k}}$ test vectors are required to test the circuit. Test vector set is derived by filtering the high fault coverage test vectors from the actual number of test vectors (here BIST analysis \& diagnosis tool is used filter six high fault coverage test vectors of s396 benchmark circuit). In order to avoid the problem called pattern minimization a technique is carried to compare the entire test vector set based on the fault detection ability, if many test vectors detects the same fault with one bit variation in 
the test vector sequence than that place is filled with ' $\mathrm{x}$ ', by this method here six test vectors are found as essential for ISCAS89 s396 benchmark circuit. When 128 test vectors are optimized to six test vectors then the test time effectively reduced to the minimum. For this s396 circuit 128 test vectors are required to test the circuit then six test vectors are filtered by using BISTAD tool. A test vector set $\mathrm{T}$ is given below:

$$
T=[T[1], T[2], T[3], T[4], T[5], T[6]]=T[5,6,11,14,21,88]
$$

These six test vectors are considered as node here, all odd value from 0 to 127 are taken in account to formulate the sequence. The odd value sequence of $(127,3)$ is $\{0,3,6,9,12,15$ ,18,21 ,24,27,30,33,36,39,42,45,48,51,54,57,60,63,66,69,72,75,78,81,84,87,90,93,96,99,1 02,105,108, $111,114,117,120,123,126,1,4,7,10,13,16,19,22,25,28,31,34,37,40,43,46,49,52$ ,55,58,61,64,67,70,73,76,79,82,85,88,91,94,97,100,103,106,109,112,115,118,121,124,12 7,2,5,8, 11,14,17,20,23,26,29,32,35,38,41,44,47,50,53,56,59,62,65,68,71,74,77,80,83,86,8 9,92,95,98,101,104,107,110,113,116,119,122,125\}.Likewise it is preceded for all possible combination i.e., $(127, \mathrm{n})$. Here $\mathrm{n}$ is the odd numbers in-between $(0$ to 127) because $\mathrm{k}=$ 128. In Table 2 decimal representation of the test vectors are given in first row, column and their location are given in $6 * 6$ matrix forms. Matrix size is equivalent to the number of test vectors in the test vector set of the concern circuit. In general for $\mathrm{k}$ inputs $2^{\mathrm{k}}-1$ matrix are required to derive $A_{\min }$ and $A_{\text {vec }}$ matrix. $A_{\min }$ and Avec are derived by finding the minimum values of a particular point for example all matrix value of 6 to 11 are compared and got 1 as minimum value which is taken for $\mathrm{A}_{\min }$ and the corresponding matrix value $\mathrm{A} 5$ is the $\mathrm{A}_{\text {vec }}$ value. Addend patterns are in the form of $2^{\mathrm{n}}+1$ i.e., $2^{1}+1=3,2^{2}+1=5, \ldots$ if the addend patterns are in the form of $2^{\mathrm{n}}+1$ then $3,5,9,17,33$ and 65 are its test pattern set.

\section{PROPOSED METHOD}

In this paper minimum spanning tree is introduced rather than Hamiltonian path (Hamiltonian path is a path which visits each vertex exactly once and also returns to the starting vertex) in the graphical construction of the c17 \& s386 benchmark circuit. Minimum spanning tree is a tree in a graph that spans all the vertices and total weight of a tree is minimal. Addend patterns are in the form of $2^{n}+1 \& 2^{n}+3$ are taken to compare the Hamiltonian path time and minimum spanning tree time cycles. 
Table 2. Amin of $c 17$ circuit with respect to $(2 n+1)$ test patterns.

\begin{tabular}{|c|c|c|c|c|c|}
\hline Amin & $\mathbf{1 1}$ & $\mathbf{1 4}$ & $\mathbf{1 7}$ & $\mathbf{2 8}$ & $\mathbf{3 1}$ \\
\hline $\mathbf{1 1}$ & $\mathrm{x}$ & 1 & 2 & 1 & 3 \\
\hline $\mathbf{1 4}$ & 10 & $\mathrm{x}$ & 1 & 5 & 1 \\
\hline $\mathbf{1 7}$ & 5 & 11 & $\mathrm{X}$ & 14 & 5 \\
\hline $\mathbf{2 8}$ & 5 & 1 & 4 & $\mathrm{x}$ & 1 \\
\hline
\end{tabular}

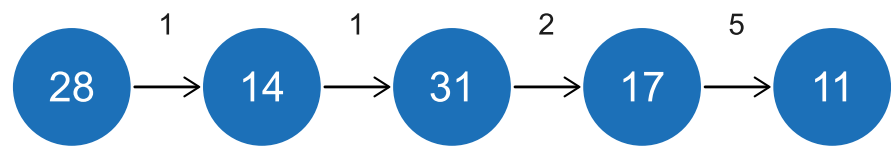

Figure 1. Hamiltonian path of $c 17$ with Addend patterns are in the form of $\left(2^{n}+1\right)$.

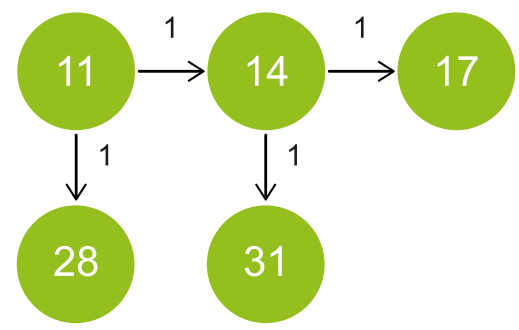

Figure 2. Minimum spanning tree of $c 17$ with Addend patterns are in the form of $\left(2^{n}+1\right)$.

The shortest Hamiltonian path for c17 circuit is $28 \rightarrow 14 \rightarrow 31 \rightarrow 17 \rightarrow 11(1,1,2,5)$ with corresponding weights and its total weight is 9 but in case of minimum spanning tree 4 time cycle are required (Figure $1 \& 2$ ). For Addend patterns are in the form of $2^{\mathrm{n}}+3$ and the Hamiltonian path through $17 \rightarrow 28 \rightarrow 11 \rightarrow 31 \rightarrow 14$ and their corresponding weights are $(1,2$, $4,3)$ totally 10 time cycles are involved whereas in minimum spanning tree with revisiting it is reduced to 7 which denotes that 14 times cycles (Figure $3 \& 4$ ) are required for testing. Table 3. Amin of c17 circuit with respect to $(2 n+3)$ test patterns.

\begin{tabular}{|c|c|c|c|c|c|}
\hline Amin & $\mathbf{1 1}$ & $\mathbf{1 4}$ & $\mathbf{1 7}$ & $\mathbf{2 8}$ & $\mathbf{3 1}$ \\
\hline $\mathbf{1 1}$ & $\mathrm{x}$ & 14 & 9 & 10 & 4 \\
\hline $\mathbf{1 4}$ & 4 & $\mathrm{x}$ & 13 & 2 & 10 \\
\hline $\mathbf{1 7}$ & 5 & 5 & $\mathrm{x}$ & 1 & 2 \\
\hline $\mathbf{2 8}$ & 2 & 10 & 4 & $\mathrm{X}$ & 13 \\
\hline $\mathbf{3 1}$ & 2 & 3 & 11 & 5 & $\mathrm{x}$ \\
\hline
\end{tabular}




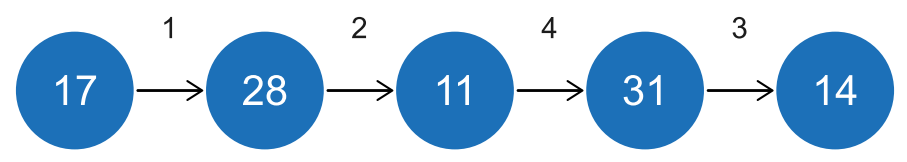

Figure 3. Hamiltonian path of $c 17$ with Addend patterns are in the form of $\left(2^{n}+3\right)$.

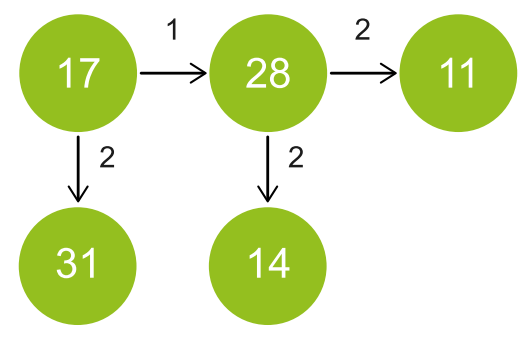

Figure 4. Minimum spanning tree of $c 17$ with Addend patterns are in the form of $\left(2^{n}+3\right)$.

Table 4. Test vector set of s396 circuit.

\begin{tabular}{|c|c|}
\hline Test Vector & Inputs [6:0] \\
\hline T1 & 0000101 \\
\hline T2 & 0000110 \\
\hline T3 & 0001011 \\
\hline T4 & 0001110 \\
\hline T5 & 0010101 \\
\hline T6 & 1010000 \\
\hline
\end{tabular}

Revisiting can reduce the testing time, here s396 benchmark circuit is taken as example which deals with 7 inputs and therefore $2^{\mathrm{k}}$ test vectors are required to test the circuit i.e., 128 test vectors. $\mathrm{A}_{\min }$ and $\mathrm{A}_{\text {vec }}$ are tabulated to derive the s396 circuit's graphical representation. All odd value sequence from 0 to 127 is taken in account for $\mathrm{A}_{\text {min }}$ and Avec calculation. The shortest Hamiltonian path for s396 circuit is $6 \rightarrow 21 \rightarrow 88 \rightarrow 5 \rightarrow 11 \rightarrow 14(1,5,3,2$, 1) with corresponding weights and its total weight is 12 but in case of minimum spanning tree 11 time cycle are required (Figure 2). Figure 3 shows the graphical representation of s386 circuit where $\mathrm{A}_{\min } \&$ Avec are derived with the consolidation of 64 matrices (all odd sequence from 0 to 127$)$. 


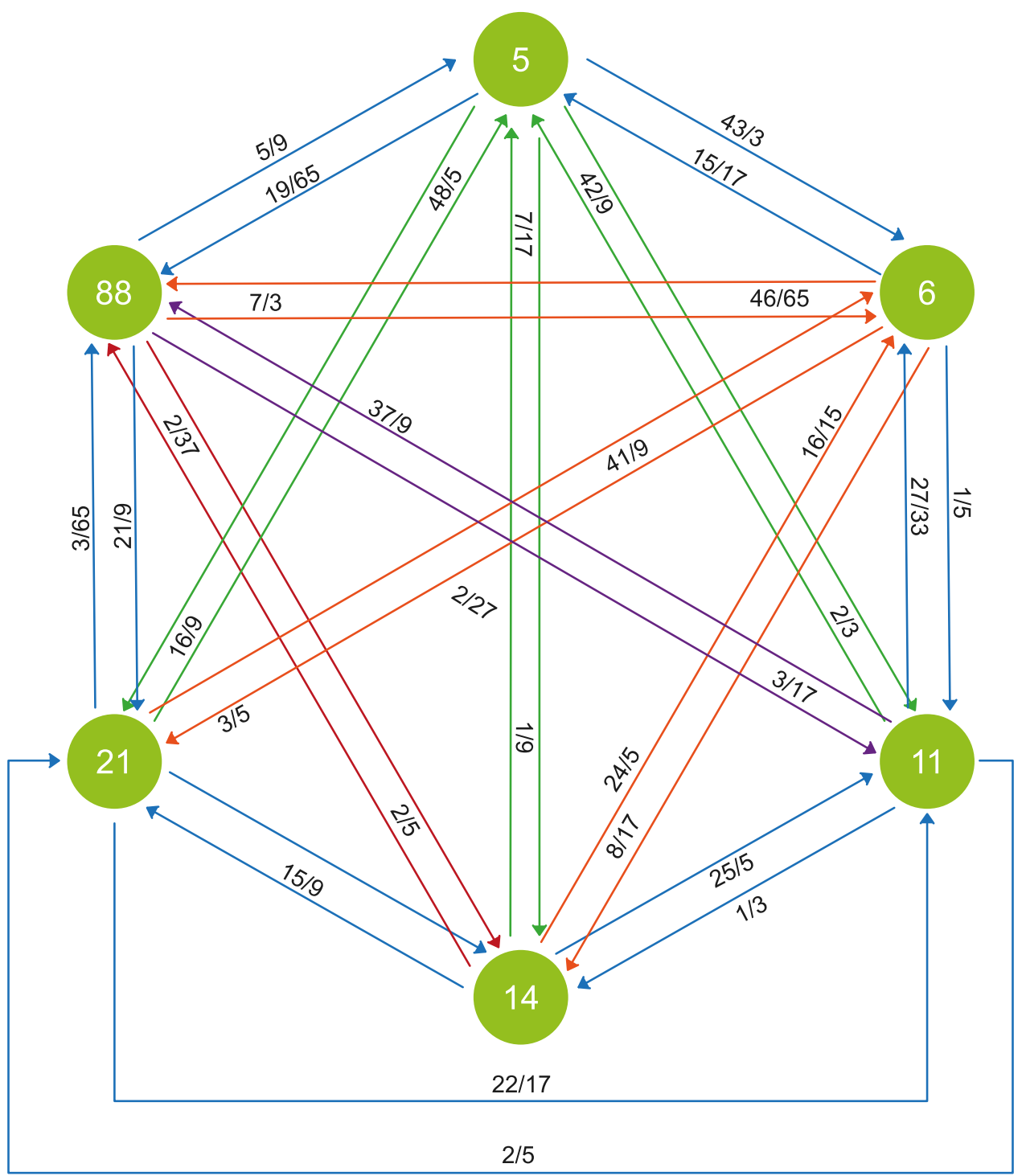

Figure 5. Graphical representation of s396 circuit.

Table 5. $A_{\min }$ of $s 386$ circuit with respect to $\left(2^{n}+1\right)$ test patterns.

\begin{tabular}{|c|c|c|c|c|c|c|}
\hline $\mathbf{A}_{\mathbf{m i n}}$ & $\mathbf{5}$ & $\mathbf{6}$ & $\mathbf{1 1}$ & $\mathbf{1 4}$ & $\mathbf{2 1}$ & $\mathbf{8 8}$ \\
\hline $\mathbf{5}$ & $\mathrm{x}$ & 43 & 2 & 1 & 16 & 19 \\
\hline $\mathbf{6}$ & 15 & $\mathrm{x}$ & 1 & 8 & 3 & 7 \\
\hline $\mathbf{1 1}$ & 42 & 27 & $\mathrm{x}$ & 1 & 2 & 37 \\
\hline $\mathbf{1 4}$ & 7 & 24 & 25 & $\mathrm{x}$ & 15 & 2 \\
\hline $\mathbf{2 1}$ & 48 & 41 & 22 & 57 & $\mathrm{x}$ & 3 \\
\hline $\mathbf{8 8}$ & 5 & 46 & 3 & 6 & 21 & $\mathrm{x}$ \\
\hline
\end{tabular}


Table 6. $A_{\min }$ of $s 386$ circuit with respect to $\left(2^{n}+3\right)$ test patterns.

\begin{tabular}{|c|c|c|c|c|c|c|}
\hline $\mathbf{A}_{\min }$ & $\mathbf{5}$ & $\mathbf{6}$ & $\mathbf{1 1}$ & $\mathbf{1 4}$ & $\mathbf{2 1}$ & $\mathbf{8 8}$ \\
\hline $\mathbf{5}$ & $\mathrm{x}$ & 11 & 2 & 53 & 48 & 17 \\
\hline $\mathbf{6}$ & 21 & $\mathrm{x}$ & 1 & 24 & 3 & 6 \\
\hline $\mathbf{1 1}$ & 46 & 73 & $\mathrm{x}$ & 33 & 2 & 7 \\
\hline $\mathbf{1 4}$ & 13 & 24 & 23 & $\mathrm{x}$ & 1 & 30 \\
\hline $\mathbf{2 1}$ & 16 & 59 & 18 & 11 & $\mathrm{x}$ & 1 \\
\hline $\mathbf{8 8}$ & 9 & 58 & 49 & 18 & 27 & $\mathrm{x}$ \\
\hline
\end{tabular}

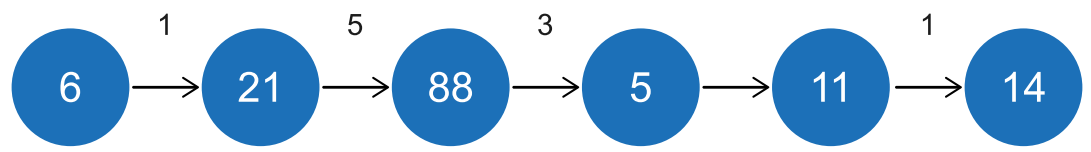

Figure 6. Hamiltonian path of $s 386$ with Addend patterns are in the form of $\left(2^{n}+1\right)$.

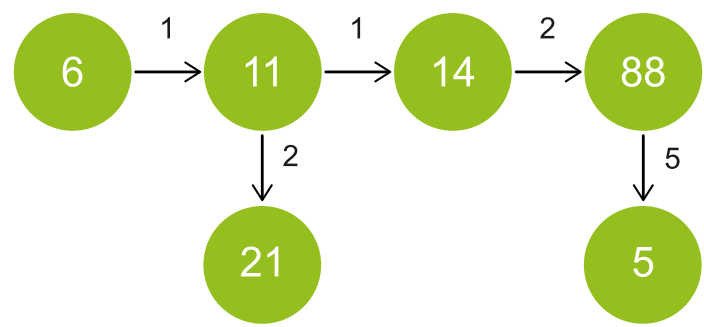

Figure 7. Minimized time spanning tree of s396 with Addend patterns are in the form of $\left(2^{n}+1\right)$.

For Addend patterns are in the form of $2^{\mathrm{n}}+3$ the Hamiltonian path through $14 \rightarrow 21 \rightarrow$ $88 \rightarrow 5 \rightarrow 6 \rightarrow 11$ and their corresponding weights are $(1,1,9,11,1)$ totally 23 time cycles are involved whereas in minimum spanning tree with revisiting it is reduced to 14 which denotes that 14 times cycles (Figure 3) are required for testing.

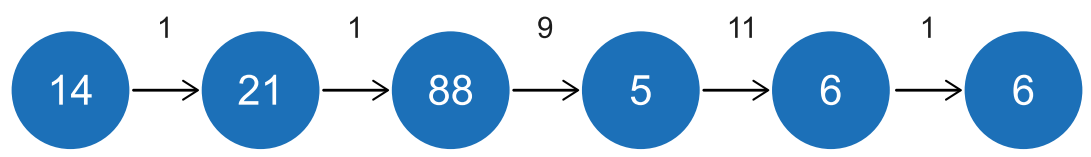

Figure 8. Hamiltonian path of $s 386$ with Addend patterns are in the form of $\left(2^{n}+3\right)$.

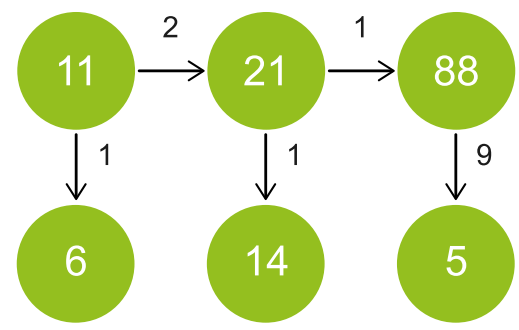

Figure 9. Minimized time spanning tree of s396 with Addend patterns are in the form of $\left(2^{n}+3\right)$. 


\section{COMPARISON}

\begin{tabular}{|c|c|c|c|}
\hline Benchmark circuit & $\begin{array}{c}\text { Addend pattern } \\
\text { form }\end{array}$ & $\begin{array}{c}\text { Hamiltonian path Time cycle } \\
\text { requirement }\end{array}$ & $\begin{array}{c}\text { Minimum spanning tree Time } \\
\text { cycle requirement }\end{array}$ \\
\hline \multirow{2}{*}{ C17 } & $2^{\mathrm{n}}+1$ & 9 & 4 \\
\hline $2^{n}+3$ & 10 & 7 \\
\hline \multirow{2}{*}{ S386 } & $2^{n}+1$ & 12 & 11 \\
\hline $2^{n}+3$ & 23 & 14 \\
\hline
\end{tabular}

Table 7. Comparison table for time cycle involvement in Hamiltonian path, minimum spanning tree with revisiting nodes.

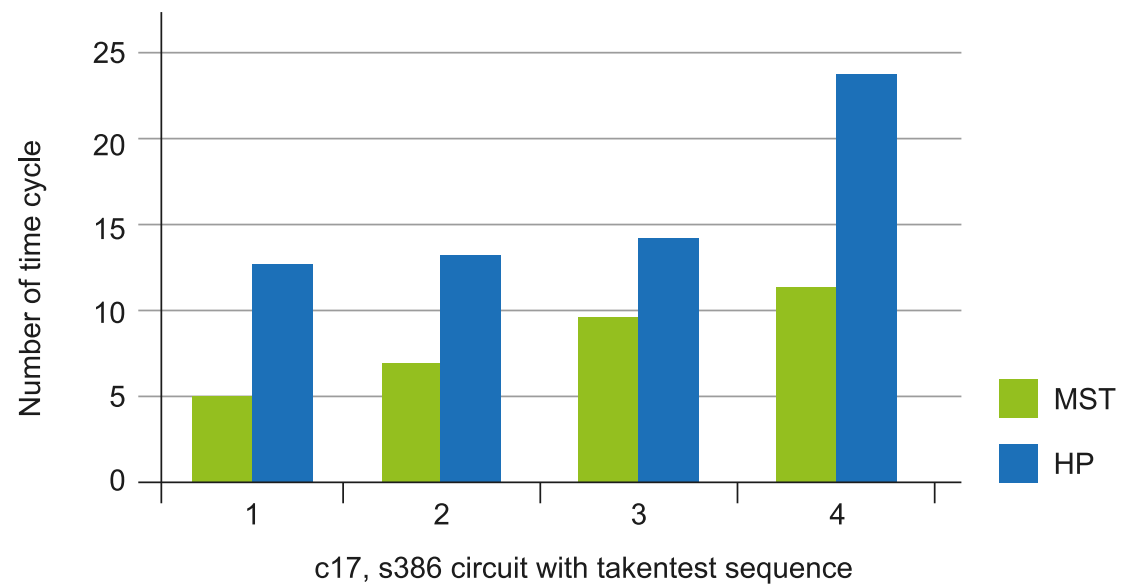

Figure 10. Graph for time cycle involvement in Hamiltonian path, minimum spanning tree with revisiting nodes.

\section{CONCLUSION}

In this paper we have presented a graph theory concept called minimum spanning tree with revisiting nodes instead of Hamiltonian path for c17 \& s396 benchmark circuit which results in optimized test time. Result shows that minimum spanning tree with revisiting the nodes will reduce the time cycle for testing. The above mentioned Table $7 \&$ Figure 10 shows that minimum spanning tree effectively reduces the number of test time cycles for testing. In future it can be implemented to test nano memories. 


\section{REFERENCES}

Alpert, G. J., Chow, W. K., Han, K., Kahng, A. B., Li, Z., Liu, D., \& Venkatesh, S. (2018, March). Prim-Dijkstra Revisited: Achieving Superior Timing-driven Routing Trees. In Proceedings of the 2018 International Symposium on Physical Design (pp. 10-17). ACM.

Bhakthavatchalu, R., Krishnan, S., Vineeth, V., \& Devi, M. N. (2014, July). Deterministic seed selection and pattern reduction in Logic BIST. In 18th International Symposium on VLSI Design and Test (pp. 1-2). IEEE.

Biswas, S., Das, S. R., \& Petriu, E. M. (2006). Space compactor design in VLSI circuits based on graph theoretic concepts. IEEE Transactions on Instrumentation and Measurement, 55(4), 1106-1118. https://doi.org/10.1109/TIM.2006.876523

Bryant, R. E. (1986). Graph-based algorithms for boolean function manipulation. IEEE Transactions on Computers, 100(8),677-691.https://doi.org/10.1109/TC.1986.1676819

Ghandra, A., \& Ghakrabarty, K. (2003). A unified approach to reduce SOC test data volume, scan power and testing time. IEEE transactions on computer-aided design of integrated circuits and systems, 22(3), 352-363. https://doi.org/10.1109/TCAD.2002.807895

Ghloupek, M., Novak, O., \& Jenicek, J. (2012, April). On test time reduction using pattern overlapping, broadcasting and on-chip decompression. In 2012 IEEE 15th International Symposium on Design and Diagnostics of Electronic Circuits ES Systems (DDECS) (pp. 300-305). IEEE.

Ghloupek, M., Novak, O., \& Jenicek, J. (2012, April). On test time reduction using pattern overlapping, broadcasting and on-chip decompression. In 2012 IEEE 15th International Symposium on Design and Diagnostics of Electronic Circuits \& Systems (DDECS) (pp. 300-305). IEEE.

Das, S. R., Hossain, A., Biswas, S., Petriu, E. M., Assaf, M. H., Jone, W. B., \& Sahinoglu, M. (2008). On a new graph theory approach to designing zero-aliasing space compressors for built-in self-testing. IEEE Transactions on Instrumentation and Measurement, 57(10), 2146-2168. https://doi.org/10.1109/TIM.2007.910004 
Flores, P., Neto, H., Chakrabarty, K., \& Marques-Silva, J. (1999, May). Test pattern generation for width compression in BIST. In ISCAS'99. Proceedings of the 1999 IEEE International Symposium on Circuits and Systems VLSI (Cat. No. 99CH36349) (Vol. 1, pp. 114-118). IEEE.

Girard, P., Nicolici, N., \& Wen, X. (Eds.). (2010). Power-aware testing and test strategies for low power devices. Springer Science \& Business Media.

Gogoi, B., \& Kalita, B. (2014). Algorithm to color a Circuit Dual Hypergraph for VLSI Circuit. International Journal of Computer Science and Information Technologies, 5(4), $5047-$ 5052. http://ijcsit.com/docs/Volume\%205/vol5issue04/ijcsit2014050447.pdf

Kim, H. S., \& Kang, S. (2006). Increasing encoding efficiency of LFSR reseeding-based test compression. IEEE Transactions on Computer-Aided Design of Integrated Circuits and Systems, 25(5), 913-917. https://doi.org/10.1109/TCAD.2005.855977

Lai, W. J., Kung, C. P., \& Lin, G. S. (1993, February). Test time reduction in scan designed circuits. In 1993 European Conference on Design Automation with the European Event in ASIC Design (pp. 489-493). IEEE.

Larsson, E. (2006). Introduction to advanced system-on-chip test design and optimization (Vol. 29). Springer Science \& Business Media.

Liang, W., Zhang, D., You, Z., Li, W., \& Hosam, O. (2013). A survey of techniques for VLSI IP protection. Information Technology Journal, 12(12), 2324-2332. https:// doi.org/10.3923/itj.2013.2324.2332

Lien, W. G., Lee, K. J., Hsieh, T. Y., \& Ghakrabarty, K. (2014). Efficient LFSR reseeding based on internal-response feedback. Fournal of Electronic Testing, 30(6), 673685. https://doi.org/10.1007/s10836-014-5482-4

Magos, D., Voyiatzis, I., \& Tarnick, S. (2010). An Accumulator-Based Test-Per-Clock Scheme. IEEE Transactions on Very Large Scale Integration (VLSI). Systems, 19(6), 1090-1094. https://doi.org/10.1109/TVLSI.2010.2043452 
Manich, S., Garcia-Deiros, L., \& Figueras, J. (2007). Minimizing test time in arithmetic test-pattern generators with constrained memory resources. IEEE Transactions on Computer-Aided Design of Integrated Circuits and Systems, 26(11), 2046-2058. https://doi. org/10.1109/TCAD.2007.906465

Nicolici, N., \& Al-Hashimi, B. (2003). Power-constrained testing of VLSI circuits (p. 178). Kluwer Academic Publishers.

Pathak, V. K., \& Pathak, V. K. (2016). Design of Input Vector Monitoring Concurrent BIST based Architecture for 4-Bit Multiplier. International Journal of Computer Applications, 153(5), 19-24. https://pdfs.semanticscholar. org/9c0e/229b71f418dabcb96b54af0e8474116c9370.pdf

Sakurai, T., \& Newton, A. R. (1990). Alpha-power law MOSFET model and its applications to CMOS inverter delay and other formulas. IEEE Journal of solid-state circuits, 25(2), 584-594. https://doi.org/10.1109/4.52187

Shanmugasundaram, P., \& Agrawal, V. D. (2011, May). Dynamic scan clock control for test time reduction maintaining peak power limit. In 29th VLSI Test Symposium (pp. 248-253). IEEE.

Shanmugasundaram, P., \& Agrawal, V. D. (2012, January). Externally tested scan circuit with built-in activity monitor and adaptive test clock. In 2012 25th International Conference on VLSI Design (pp. 448-453). IEEE.

Tehranipoor, M., Nourani, M., \& Chakrabarty, K. (2005). Nine-coded compression technique for testing embedded cores in SoCs. IEEE transactions onvery largescaleintegration (VLSI) systems, 13(6), 719-731. https://doi.org/10.1109/TVLSI.2005.844311

Tharakan, A. S., \& Mathew, B. K. (2015). Design and Implementation of an On-Chip Test Generation Scheme Based on Reconfigurable Run-Time Programmable and Multiple Twisted-Ring Counters. Procedia Computer Science, 46, 1409-1416. https:// doi.org/10.1016/j.procs.2015.02.059 
Venkataramani, P., Sindia, S., \& Agrawal, V. D. (2014). A test time theorem and its applications. Fournal of Electronic Testing, 30(2), 229-236. https://doi.org/10.1007/ s10836-014-5447-7

Voyiatzis, I. (2005). Test vector embedding into accumulator-generated sequences: A linear-time solution. IEEE Transactions on Computers, 54(4), 476-484. https://doi. org/10.1109/TC.2005.69

Voyiatzis, I. (2006, September). Accumulator-based compression in symmetric transparent RAM BIST. In International Conference on Design and Test of Integrated Systems in Nanoscale Technology, 2006. DTIS 2006. (pp. 273-278). IEEE.

Voyiatzis, I. (2008, March). On reducing aliasing in accumulator-based compaction. In 2008 3rd International Conference on Design and Technology of Integrated Systems in Nanoscale Era (pp. 1-12). IEEE.

Zhou, B., Ye, Y. Z., Li, Z. L., Wu, X. C., \& Ke, R. (2009, March). A new low power test pattern generator using a variable-length ring counter. In 2009 10th International Symposium on Quality Electronic Design (pp. 248-252). IEEE. 